\title{
An automatic differentiation-based gradient method for inversion of the shear wave equation in magnetic resonance elastography: specific application in fibrous soft tissues
}

\author{
Simon Chatelin ${ }^{1}$, Isabelle Charpentier ${ }^{1}$, Nadège Corbin ${ }^{1}$, Laurence Meylheuc ${ }^{1}$ and \\ Jonathan Vappou ${ }^{1}$ \\ ${ }^{1}$ ICube, University of Strasbourg, CNRS, IHU Strasbourg, France $\quad$ E-mail: schatelin@ unistra.fr
}

\begin{abstract}
Quantitative and accurate measurement of in vivo mechanical properties using dynamic elastography has been the scope of many research efforts over the past two decades. Most of the shear-wave-based inverse approaches for magnetic resonance elastography (MRE) make the assumption of isotropic viscoelasticity. In this paper, we propose a quantitative gradient method for inversion of the shear wave equation in anisotropic media derived from a full waveform description using analytical viscoelastic Green formalism and automatic differentiation. The abilities and performances of the proposed identification method are first evaluated on numerical phantoms calculated in a transversely isotropic medium, and subsequently on experimental MRE data measured on an isotropic hydrogel phantom, on an anisotropic cryogel phantom and on an ex vivo fibrous muscle. The experiments are carried out by coupling circular shear wave profiles generated by acoustic radiation force and MRE acquisition of the wave front. Shear modulus values obtained by our MRE method are compared to those obtained by rheometry in the isotropic hydrogel phantom, and are found to be in good agreement despite non-overlapping frequency ranges. Both the cryogel and the ex vivo muscle are found to be anisotropic. Stiffness values in the longitudinal direction are found to be 1.8 times and 1.9 times higher than those in the transverse direction for the cryogel and the muscle, respectively. The proposed method shows great perspectives and substantial benefits for the in vivo quantitative investigation of complex mechanical properties in fibrous soft tissues.
\end{abstract}

Keywords: magnetic resonance elastography, anisotropy, inverse problems, automatic differentiation, Green formalism

\section{Introduction}

Over the past two decades, dynamic elasticity imaging (dynamic elastography) has emerged as a virtual palpation tool allowing for a semi-quantitative investigation of the in vivo mechanical response of biological soft tissue by the measurement of the viscoelastic mechanical properties of the organ under study. Classically, the three main steps of such methods are [Vappou et al. 2012]:

(i) The generation of either harmonic or transient shear waves in the organ under study,

(ii) The measurement of the displacement field using ultrasound ([Fatemi and Greenleaf 1998, Sarvazyan et al. 1998, Sandrin et al. 2003, Bercoff et al. 2004]) or Magnetic Resonance Imaging (MRE, [Muthupillai et al. 1995]),

(iii) The identification of the mechanical properties (usually the shear modulus).

This last step requires the development of specific algorithms coupling wave physics and biomechanics. The local viscoelastic parameters may be estimated through the displacement field resulting from the shear wave propagation [Catheline et al. 2004]. In a uniform, homogeneous, purely linear elastic medium, the wave equation is written as:

$$
\rho \frac{\partial^{2}}{\partial t^{2}} \boldsymbol{U}-(\lambda+2 \mu) \nabla^{2} \boldsymbol{U}=0,
$$

$\boldsymbol{U}$ being the displacement vector, $t$ the time and $\rho$ the density of the medium, $\boldsymbol{F}$ the body-forces, and $\lambda$ and $\mu$ the first and the second Lamé coefficients, respectively. In MRE, algorithms such as the Local Frequency Estimation (LFE), the Phase Gradient (PG) method [Manduca et al. 1996, 2001] and the Direct Inversion (DI) of the shear wave equation [Oliphant et al. 2001] have been proposed for the identification of the mechanical properties from the displacement field. In 1999, [Van Houten et al. 1999] suggested to use a finite elementbased reconstruction operating on small overlapping subzones to determine elastic properties in a heterogeneous medium. 
The inverse elasticity problem can be solved as a non-linear optimization problem. This approach aims at finding the distribution of mechanical properties (usually the shear modulus) that minimizes the difference between the measured and predicted displacement fields (computed with either an analytical formulation or a finite element method or a finite difference method). The gradient methods are quantitative and robust [Arnal et al. 2013]. The use of an adjoint method has been proposed in [Oberai et al. 2003] for identification of heterogeneous shear modulus maps in an incompressible isotropic medium using a finite element scheme of wave propagation and one of the components of the displacement field. More recently, a gradient approach has been adopted for breast tumor characterization using MRE [Wang et al. 2009].

Although many tissues (such as skeletal muscle, cardiac muscle, kidney or brain) exhibit anisotropic mechanical properties, i.e. properties being directionally dependent [Fung 1993], few in vivo studies have investigated the anisotropic behavior of biological soft tissues using elastography. Ultrasound shear wave elastography (SWE) has been used for anisotropic in vivo elasticity measurements of fibrous tissues, such as myocardium [Couade et al. 2011, Lee et al. 2012, Song et al. 2014, Correia et al. 2014], skeletal muscles [Gennisson et al. 2003, 2005, 2010], kidney [Amador et al. 2011, Gennisson et al. 2012], tendons [Brum et al. 2013] or arteries [Shcherbakova et al. 2014]. However, the actual SWE techniques allow for shear wave propagation in two opposite directions only. Thus, the investigation of the anisotropic stiffness requires acquisitions with different orientations for the probe [Lee et al. 2012, Chatelin et al. 2015]. Most of the MRE techniques consider the organ as isotropic. First MRE anisotropic measurements have been proposed in [Sinkus et al. 2005] for breast tissue using optimization techniques to identify the principal axis of a transversely isotropic material model. Anisotropic viscoelasticity has also been investigated in [Papazoglou et al. 2006, Sack et al. 2009, Klatt et al. 2010] in human skeletal muscle by analyzing the directional dependence of the shear wave pattern assuming an incompressible and transverse isotropic model of elasticity. As discussed in [Bensamoun et al. 2006, Ringleb et al. 2007], MRE allows for the detection of differences in the orientation as well as of differences in the muscle fiber. Under the a priori knowledge of uniaxial alignment of fibers [Namani et al. 2009], the anisotropic elasticity has been investigated in a fibrin gel by generating shear waves either parallel or perpendicular to the fibers. Recently, studies have shown the major physiological role played by the mechanical anisotropy in fibrous tissues [Ringleb et al. 2007, Bensamoun et al. 2007, McCullough et al. 2011]. The possibility of coupling MRE reconstruction and Diffusion Tensor Imaging (DTI, providing estimation of the local fiber orientation from MRI of the constrained water Brownian diffusion) has been proposed in [Green et al. 2013, Qin et al. 2013] to investigate the anisotropic elasticity of ex vivo soft tissues, again under the assumption of one predominant fiber direction. It is worth noting that all of these approaches require either a local transverse isotropic modeling of the medium or complementary information on the fibers orientation in the tissue. These limitations are mainly due to the fact that, in all these approaches, the source for the generation of waves is insufficiently known.

Measurements of the properties of muscle may shed light on the effects of pathologies that change muscle fiber composition, like hyperthyroidism [Bensamoun et al. 2007], and help improve our approach for treating individuals affected by these disorders. It is practical to start using MRE to answer specific clinical questions in pathologies that effect muscles. For example, MRE can be used to evaluate treatments for muscles altered by pathology (e.g., hyperthyroidism and stroke) and injured muscle (e.g., muscle atrophy caused by disuse).

Mechanical properties may be an efficient biomarker for pathologies that affect the fiber structure of the organs. Measuring the mechanical anisotropy has been shown to be particularly interesting and relevant for diagnostic assessment and clinical treatment evaluation in different disorders that alter fibers, such as muscular atrophy (Ringleb et al 2007), poliomyelitis, paraplegia (Basford et al 2002), cardiac hypertrophy, cardiomyopathy, post-infarction remodeling (Lee et al 2012), renal fibrosis (Arndt et al 2010) and nephrocalcinosis (Shah et al 2004). The development of new robust tools for quantitative dynamic elastography in anisotropic tissues is therefore particularly relevant in the field of in vivo biomechanical characterization. In this study, we develop a new MRE protocol for elasticity imaging and identification in fibrous tissues combining two approaches, detailed as follows.

(i) First, shear waves are generated in a non-invasive manner applying a localized nearly-punctual source on the tissue. As in [Wu et al. 2000] and [Souchon et al. 2008], we associate the Acoustic Radiation Force (ARF) from a MR-compatible High Intensity Focalized Ultrasound (HIFU) probe as a source for remote generation of shear waves, with MRE for the measurement of the displacement wave field. By alternating emission and non-emission periods of the focused ultrasounds, the ARF is generated with a periodic square time-profile (with frequency $f_{0}$ ). The resulting shear waves are pseudo-harmonic (with frequency $f_{0}$ ). The mechanical excitation is synchronized on the MRE sequence. 
(ii) Second, we develop a gradient-based data assimilation approach to solve the inverse problem in anisotropic viscoelastic soft tissues. The original solution proposed in this article to investigate anisotropy consists of converting the shear wave field from 2DCartesian to polar coordinates, restricting the displacement field to specific directions of space and then identifying the analytical description using the uniaxial viscoelastic Green formalism. The model is differentiated using an automatic differentiation (AD) tool to compute accurate gradients (Griewank and Walther 2008). The gradient method is applied for the identification of the shear elasticity in 36 angular directions of space from a single MRE acquisition of the displacement field. The use of a gradient-based data assimilation method together with AD shows a great potential for MRE reconstructions, even with no assumption about the mechanical anisotropy.

This MRE protocol is first evaluated on numerical isotropic phantoms, and then on experimental MRE data (elastic hydrogel phantom, anisotropic cryogel phantom and ex vivo muscle).

\section{Material and methods}

In the present section, the formulation of the forward model and the inverse problem for shear wave propagation in anisotropic media are presented and assessed using simulated data. The experimental MRE protocol used on the gels and on the ex vivo muscle is presented.

\subsection{Formulation of the model}

In a bounded domain $\Omega$, the small displacement field $\boldsymbol{U}$ in a homogeneous elastic compressible medium with no body-force satisfies the following equations:

$$
\left\{\begin{array}{c}
\rho \frac{\partial^{2}}{\partial t^{2}} \boldsymbol{U}(\boldsymbol{x}, t)-(\lambda+2 \mu) \nabla^{2} \boldsymbol{U}(\boldsymbol{x}, t)=0 \\
\boldsymbol{U}\left(\boldsymbol{x}, t=t_{0}\right)=\boldsymbol{U}_{\mathbf{0}} \quad \text { in } \Omega
\end{array}\right.
$$

where $\boldsymbol{x}=(x, y, z), t, t_{0}$ and $\boldsymbol{U}_{\boldsymbol{0}}$ are the Cartesian coordinates, the time, the initial time and the initial displacement, respectively, and $\lambda$ and $\mu$ are the Lamé coefficients. Our approach is based on the identification of shear wave displacement profiles around the focal spot that can be seen as the source of shear waves. Equation (2) is first expressed in polar coordinates ( $\mathrm{r}, \theta)$, and then in the wave equation is restricted to a specific uniaxial direction $\theta=\theta_{0}$. Equation (2) becomes:

$$
\left\{\begin{array}{c}
\rho \frac{\partial^{2}}{\partial t^{2}} U(r, t)-\frac{(\lambda+2 \mu)}{r} \frac{\partial}{\partial r}\left(r \frac{\partial}{\partial r} U(r, t)\right)=0 \\
U\left(r, t=t_{0}\right)=U_{0} \quad \text { in } \Omega
\end{array}\right.
$$

where $U(\mathrm{r}, \mathrm{t})=U\left(\mathrm{r}, \theta=\theta_{0}, \mathrm{t}\right)$ is the unique component of the displacement vector, and $\mathrm{r}$ the nspace coordinate of the specific observation point from the source point.

In a linear viscoelastic medium undergoing small deformations, the shear wave displacement field may be described analytically by means of the viscoelastic temporal Green formalism. To that end, we assume an infinite isotropic viscoelastic homogeneous medium. The exact Green functions provide a time-harmonic plane wave description from a source point (Aki and Richards 1980). Based on the extension to viscoelastic media from the purely elastic theory developed by Aki and Richards (1980), the isotropic viscoelastic expression used in the temporal domain for the forward problem has been proposed in three dimensions by Bercoff et al (2004). By adaptation to a uniaxial wave propagation, the calculation of the $1 \mathrm{D}$ displacement vector $U(\mathrm{r}, \mathrm{t})$ from one source point is written in the temporal domain by:

$$
U(\mathrm{r}, \mathrm{t})=\sum_{l} \frac{\mathrm{F}_{l}}{4 \pi \rho}\left\{G_{k l}^{(P)}(\mathrm{r}, \mathrm{t})+G_{k l}^{(S)}(\mathrm{r}, \mathrm{t})+G_{k l}^{(P-S)}(\mathrm{r}, \mathrm{t})\right\}
$$

where $t$ is the time, $\mathrm{r}$ the $1 \mathrm{D}$ space coordinates of specific observation point from the source point, $\mathrm{F}_{1}$ the radiation force amplitude along the direction of propagation, $\rho$ the density. The terms $G_{k l}^{(P)}, G_{k l}^{(S)}$ and $G_{k l}^{(P-S)}$ correspond to the contribution of the P-wave (compression), S-wave (shear) and near-field term (between the $\mathrm{P}$-wave and $\mathrm{S}$-wave), respectively, and are written as:

$$
G_{k l}^{(P)}(\mathrm{r}, \mathrm{t})=\frac{g_{k} g_{l}}{r c_{p} \sqrt{2 \pi \eta_{p} t}} e^{-\frac{\left(t-\tau^{(1)}\right)^{2} c_{P}^{2}}{2 \eta_{p} t}},
$$




$$
\begin{aligned}
& G_{k l}^{(S)}(\mathrm{r}, \mathrm{t})=\frac{g_{k} g_{l}}{r c_{s} \sqrt{2 \pi \eta_{s} t}} e^{-\frac{\left(t-\tau^{(2)}\right)^{2} c_{S}^{2}}{2 \eta_{s} t}}, \\
& G_{k l}^{(P-S)}(\mathrm{r}, \mathrm{t})=\frac{3 g_{k} g_{l}-\delta_{k l}}{\left(\tau^{(2)} c_{s}\right)^{3}}\left\{\frac{\sqrt{\eta_{p} t / 2 \pi}}{c_{p}}\left[e^{-\frac{t^{2} c_{P}^{2}}{2 \eta_{p} t}}-e^{-\frac{\left(t-\tau^{(1)}\right)^{2} c_{P}^{2}}{2 \eta_{p} t}}\right]+\frac{t}{2}\left[\operatorname{Erf}\left(\frac{t c_{p}}{\sqrt{2 \pi \eta_{p} t}}\right)-\right.\right. \\
& \left.\left.\operatorname{Erf}\left(\frac{\left(t-\tau^{(1)}\right) c_{p}}{\sqrt{2 \pi \eta_{p} t}}\right)\right]+\frac{\sqrt{\eta_{s} t / 2 \pi}}{c_{S}}\left[e^{-\frac{t^{2} c_{s}^{2}}{2 \eta_{s} t}}-e^{-\frac{\left(t-\tau^{(2)}\right)^{2} c_{s}^{2}}{2 \eta_{s} t}}\right]+\frac{t}{2}\left[\operatorname{Erf}\left(\frac{t c_{s}}{\sqrt{2 \pi \eta_{s} t}}\right)-\operatorname{Erf}\left(\frac{\left(t-\tau^{(2)}\right) c_{s}}{\sqrt{2 \pi \eta_{s} t}}\right)\right]\right\} .
\end{aligned}
$$

where $\operatorname{Erf}$ is the error function. $\eta_{P}$ and $\eta_{S}$ correspond to the dynamic viscosity of the P- and S-wave, respectively. The travel times of the compressional $\mathrm{P}$ - and shear $\mathrm{S}$ - wave are respectively defined by:

$$
\tau^{(1)}=\frac{r}{c_{p}}, \quad \tau^{(2)}=\frac{r}{c_{s}} .
$$

The polarization vector is defined by: $\boldsymbol{g}=\left[\begin{array}{l}g_{1} \\ g_{2} \\ g_{3}\end{array}\right]$.

We consider $x$ and $\mathrm{r}$ as the polarization and propagation direction of the shear waves, respectively. The coordinates of the unit direction vector from the source to the observation point are denoted by $g_{m}=x_{m} / r$ for $m=1, \ldots, 3 . \lambda=\rho\left(c_{P}^{2}-2 c_{S}^{2}\right) \approx \rho c_{P}^{2}$ and $\mu=\rho c_{s}^{2}$ are the first and second Lame coefficients, respectively. $c_{P}$ and $c_{S}$ correspond to the celerity of the $\mathrm{P}$ - and $\mathrm{S}$-waves. In the present study, we consider shear waves polarized along the $x$-axis and we focus on the identification of $\mu$. The displacement field $U(\mathrm{r}, \mathrm{t})$ is then convolved by a harmonic sinusoidal function with a frequency $f_{0}$. It corresponds to the simulation of the profile resulting from the fundamental component of the experimental input force field $\boldsymbol{F}(\boldsymbol{x})$ at the frequency $f_{0}$.

\subsection{AD-based gradient method for the inverse problem}

The objective of the inverse problem is to estimate the values of the stiffness $\mu$ from the measurement of the observed shear wave displacement field $U^{o}$. While different parameters of the wave propagation model can be identified, we focus in this study in the estimation of the stiffness $\mu$. Consequently, the other mechanical parameters are fixed. The density and the P-wave celerity are fixed to $1038.0 \mathrm{~g} . \mathrm{cm}^{-3}$ and $1538 \mathrm{~m} . \mathrm{s}^{-1}$, respectively. Since the medium is considered as purely elastic, dynamic viscosity is fixed to $0 \mathrm{~Pa}$. These parameters correspond to an incompressible medium with a Poisson's ratio close to 0.5 for typical stiffness values found in soft tissues.

The shear wave displacement field $U^{o}$ is introduced into the identification process by means of the cost function $J$ such that:

$$
J(U(\mu))=\left\|\alpha U(\mu)-U^{o}\right\|^{2}
$$

measures the misfit between the forward model $U$ and the data $U^{o}$. In Equation $8, \alpha$ is a dimensionless constant amplitude factor corresponding to the ratio between the maximum values of the forward model and of the data. Let $\mu^{*}$ be the optimal stiffness parameter such that the solution of Equation 4 fits at best the data $U^{o}$. Under the differentiability assumption, the solution $\mu^{*}$ of the minimization problem satisfies:

$$
\begin{aligned}
\text { Find } \mu^{*} & \in \mathbf{M} \text { such that } \\
\min (J(U(\mu))) & =\min (J \circ U)(\mu)=\mu^{*}
\end{aligned}
$$

This problem can also be written as:

$$
\text { Find } \mu^{*} \in \mathbf{M} \text { such that } \nabla(J \circ U)\left(\mu^{*}\right)=0
$$

$\nabla(J \circ U)(\mu)$ is the gradient of $J \circ U(\mu)=J(U(\mu))$ evaluated at point $\mu$, ० being the composition operator. 
Our method relies on the comparison between the simulated 1D observable U (equation (4)) and experimental data expressed in polar coordinates and restricted to specific directions i (equation (3)) in order to estimate the stiffness profiles for each direction around the focal spot. We also propose to implement the code for gradient calculations by means of AD. The proposed AD-based gradient approach presented in figure 1 comprises different phases, as detailed below.

(i) The experimental displacement field $U^{o}$ results from an experimental 2D harmonic shear wave propagation. The wave field is considered in a $(y, z)$ plane from a punctual source point (coordinates $(y=0, z=0))$ at a fixed time step $t=t_{0}$. This displacement field is converted from Cartesian coordinates to Polar coordinates. This allows to build 1D displacement fields for angles ranging from $0^{\circ}$ to $360^{\circ}$. The $1 \mathrm{D}$ data profile along the $r$-axis for a fixed $\theta=\theta_{i}$ angle and time $t=t_{0}$ is denoted by $U_{\theta_{i}}^{o}\left(r, t_{0}\right)=U^{o}\left(\theta_{i}, r, t_{0}\right)$. (ii) The key-point is in the calculation of the gradient $\nabla(J \circ U)$. We use the AD software TAPENADE 3.10 [Hascoët and Pascual 2004] to differentiate the statements of the simulation code with respect to the parameters of interest. In the present case, we use the tangent linear mode of differentiation since the number of parameters to be estimated is small. This differentiation is independent from the angle and is carried out once for all. Note that the Erf function is not recognized by TAPENADE 3.10 software and should be differentiated separately (Charpentier and Dal Cappello 2015).

(iii) For each angle $\theta_{i}$,

( $\alpha$ ) the data $U^{o}\left(\theta_{i}\right)$ is compared to the simulated field $U$ (up to the scaling coefficient $\alpha$ ) by means of a cost function $J_{\theta_{i}}$;

( $\beta$ ) we proceed to the iterative minimization of the cost function $\left(J_{\theta_{i}} \circ U\right)(\mu)$ by means of the L-BFGS-B (Limited-memory Broyden-Fletcher-Goldfarb-Shanno extended to aka Bound constraints) optimizer [Zhu et al. 1997] to determine the shear stiffness $\mu^{*}\left(\theta_{i}\right)$ in the direction $\theta_{i}$.

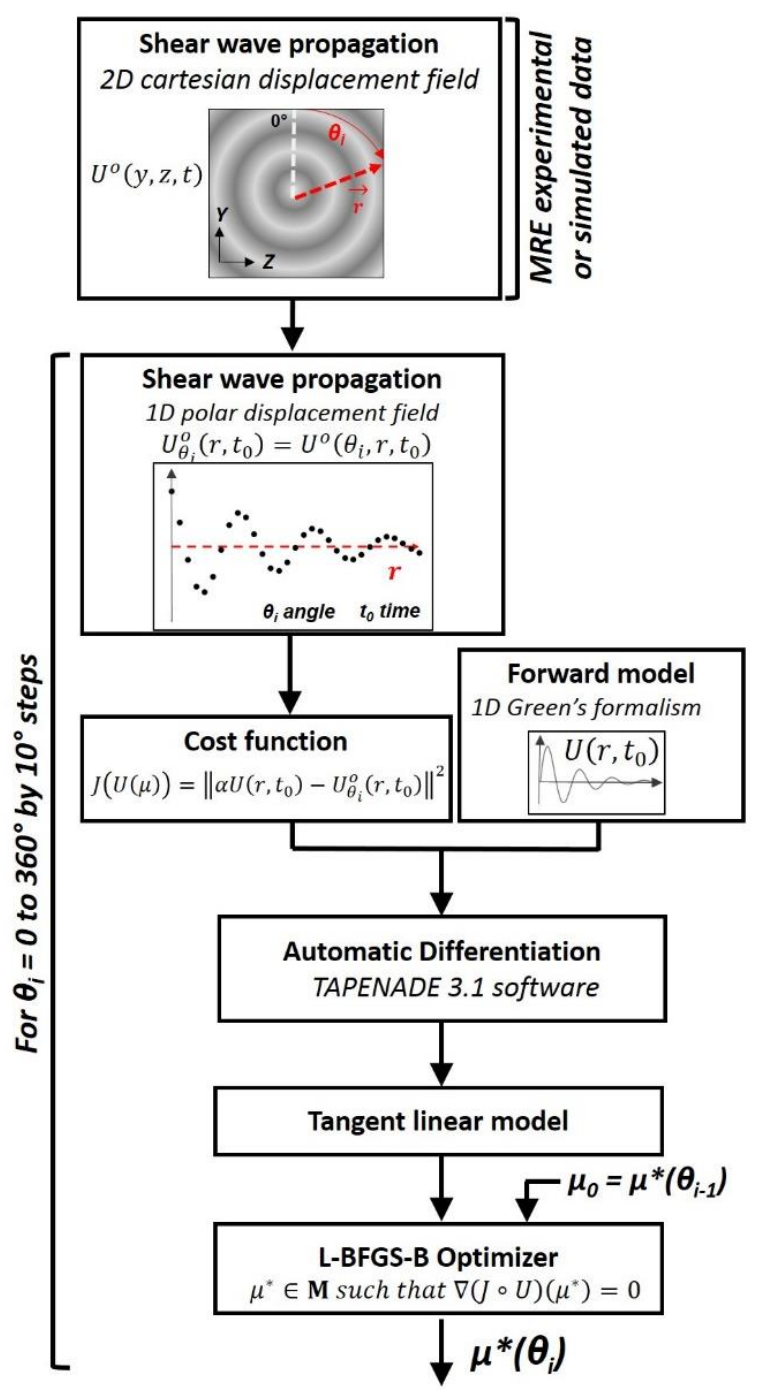

Figure 1. General overview of the algorithm. 
The numerical minimization algorithm (Equation 10) is solved by the use of the L-BFGS-B optimizer. By applying successively this protocol for $\theta_{i}=0$ to $360^{\circ}$ by $10^{\circ}$ steps, the method provides the anisotropic profile of the last computed stiffness $\mu^{*}(\theta)$ in 36 directions of space in the plane orthogonal to the ultrasonic push (focal plane). For the first angle step $\left(\theta_{i}=\theta_{0}=0^{\circ}\right)$, the initial $\mu_{0}$ parameter is estimated using a local frequency estimation (LFE)-based algorithm [Knutsson et al. 1994, Manduca et al. 2001]. For the next angles, the resulting $\mu^{*}\left(\theta_{i}\right)$ value is successively used as initial parameters $\mu_{0}$ for the $\mu^{*}\left(\theta_{i+1}\right)$ estimation to prevent divergence of the minimization process.

\subsection{Assessment in a numerical anisotropic phantom}

The proposed method for the inversion of the 1D temporal Green functions is first applied on a numerical phantom. As numerical phantom, we propose an analytical description based on the 3D Green functions in a transverse isotropic elastic soft solid [Vavryčuk 2001]. The efficiency of this description to mimic shear wave propagation in muscular tissue has been shown for elastography in 2003 by [Gennisson et al. 2003]. Further details on the analytical description for numerical anisotropic tissue mimicking phantoms can be found in the Appendix. The resulting displacement corresponds to the wave front generated by a point-source. From Equation $\mathrm{A} 3$ in the Appendix, the components $U_{\mathrm{k}}(\mathbf{x}, \mathrm{t})$ of the displacement field are then convolved by a temporal periodic square function (with $f_{0}=100 \mathrm{~Hz}$ frequency) to mimic the force signal generated experimentally by the harmonic acoustic radiation force transducer (as described in the next subsection 2.3.1). We assume this excitation results in harmonic nearly-sinusoidal shear wave field in the medium at $f_{0}$ frequency. Since shear waves are mainly polarized along the $x$-axis, we consider the $x$-component of the displacement field, i.e. $U^{o}(\mathbf{x}, t)=U_{1}(\mathbf{x}, t)$.

In this study, we consider the shear wave propagation in the $(y, z)$ plane with the main anisotropy axis (longitudinal direction) aligned with the $Y$-axis and a medium twice stiffer in the longitudinal direction than in the transverse direction. The mechanical parameters implemented in the transversely isotropic numerical phantom are summarized in table 1 . These parameters correspond to an incompressible medium with a Poisson's ratio of $v=0.4999994$. Noise was added to these numerical phantoms in order to simulate experimental MRI phase images. It has been shown that the statistical distribution of the noise in phase images with high signal-to-noise ratio (SNR > 2) could be considered as nearly Gaussian (Gudbjartsson and Patz 1995). A Gaussian-distributed noise is added on the simulated data, based on the SNR measured experimentally on MRE images. By considering the shear wave propagation in the $(\mathrm{y}, \mathrm{z})$ plane, the protocol presented in section 2.1 is applied to the numerical phantom. The results are presented in terms of the shear stiffness $\mu$ as a function of the angle $\theta$ in the $(y, z)$ plane (so that $\theta=0$ along the $y$-axis).

\begin{tabular}{c|cccc} 
& $\boldsymbol{c}_{\mathbf{1 1}}[\mathrm{kPa}]$ & $\boldsymbol{c}_{\mathbf{4 4}}[\mathrm{kPa}]$ & $\boldsymbol{c}_{\mathbf{6 6}}[\mathrm{kPa}]$ & $\boldsymbol{\rho}\left[\mathrm{g} \cdot \mathrm{cm}^{-3}\right]$ \\
\hline Numerical input parameters & $2,250.0$ & 4.0 & 2.0 & $1,038.0$ \\
\hline
\end{tabular}

Table 1. Parameters used as input for the shear wave simulations in a transverse isotropic, homogeneous, incompressible numerical phantom using Green anisotropic formalism (see Appendix).

According to the Christoffel equations, the identified anisotropic stiffness profile $\mu *(\theta)$ is then fitted by the expression for $\mu_{\text {th }}(\theta)$ (equation (12)) from a selected transverse isotropic distribution of the shear wave phase velocity $\mathrm{c}_{\mathrm{S}}(\theta)$ to obtain an estimation of the $\mathrm{c}_{44}, \mathrm{c}_{66}$ parameters (longitudinal and transverse stiffness, respectively) and main fibers orientation $\theta 0$ (Royer and Dieulesaint 2000). The identification is performed using the Curve Fitting Toolbox of the MATLAB R2015A software (Mathworks, Natick, MA, USA).

$$
\mu_{t h}(\theta)=\rho c_{S}^{2}(\theta)=c_{66} \sin ^{2}\left(\theta-\theta_{0}\right)+c_{44} \cos ^{2}\left(\theta-\theta_{0}\right),
$$

\subsection{Experimental application}

\subsubsection{Experimental MRE protocol description}

MRI and MRE acquisitions are performed on a 1.5 T MRI scanner (MAGNETOM Aera, Siemens AG, Erlangen, Germany), as presented in figure 2(A). The experimental protocol is successively applied on an isotropic gelatin hydrogel phantom, an anisotropic cryogel phantom and a fibrous beef back muscle embedded in gelatin hydrogel as presented in figures $2(\mathrm{~B})-(\mathrm{D})$, respectively. 

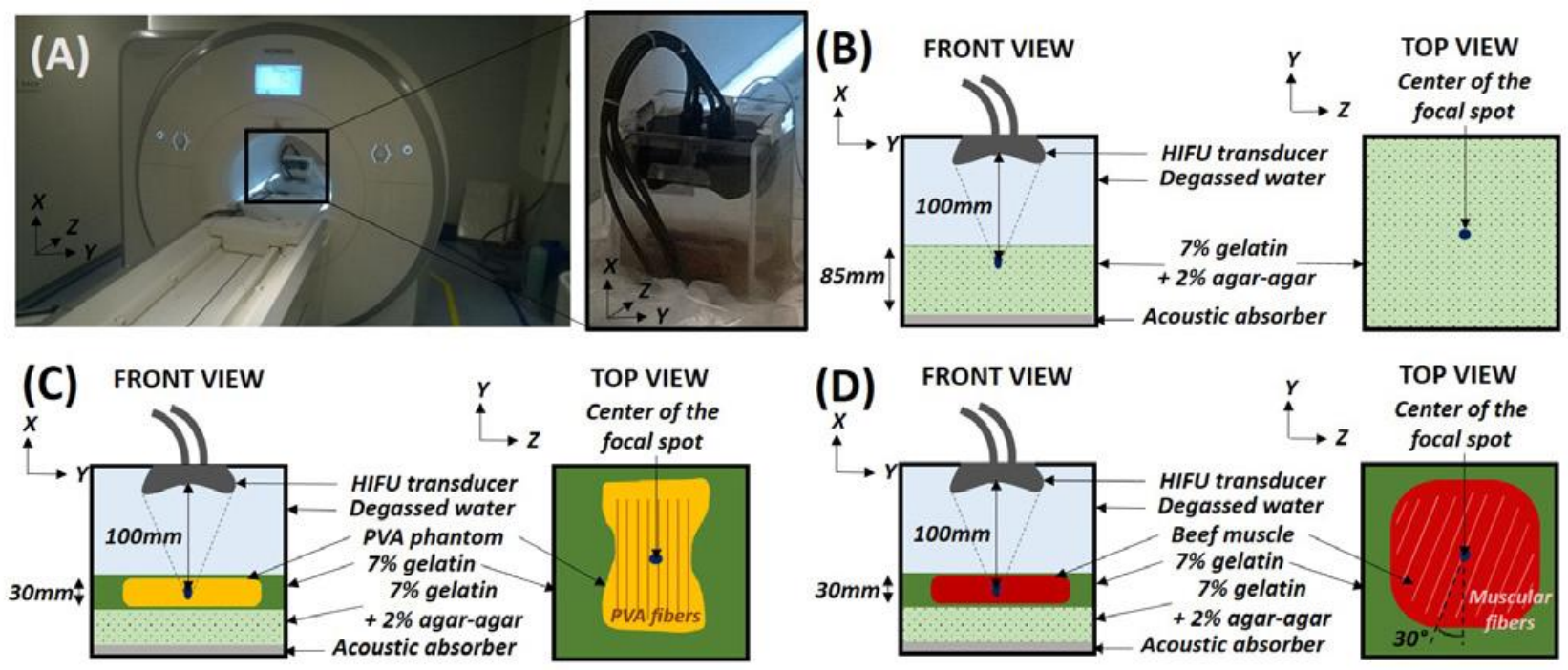

TOP VIEW

Center of the focal spot

Figure 2. General and schematic views (A) of the experimental setup for MRE measurements in an isotropic gelatin hydrogel phantom (B), in an anisotropic cryogel phantom (C) and in ex vivo fibrous beef muscle embedded in gelatin (D).

2D-MRE is performed in the coronal $(y, z)$ plane. The shear waves are generated from a 256-elements MRcompatible High Intensity Focused Ultrasound (HIFU) transducer driven at $1 \mathrm{MHz}$ (Imasonic SAS, Voray-surl'Ognon, France) immerged in degassed water as illustrated in Figure 2. A square profile of the radiation force corresponding to a mechanical excitation frequency of about $100 \mathrm{~Hz}$ is applied in the tissue by alternating periods of ARF application and periods of relaxation. The scanning parameters include the following: a spoiled gradient echo sequence with Motion-Sensitizing Gradients (MSG, $20 \mathrm{mT} / \mathrm{m}, 1$ cycle) to encode the displacement in phase images at the same frequency as the mechanical excitation (namely $100 \mathrm{~Hz}$ ), and displacement encoded in the slice $X$-direction; TE / TR $=12.12 \mathrm{~ms} / 20 \mathrm{~ms}$ at $100 \mathrm{~Hz}$; slice thickness $=10 \mathrm{~mm}$; acquisition matrix $=128 \times 128 ; \mathrm{FOV}=300 \mathrm{~mm} \times 300 \mathrm{~mm} ; 1$ slice. The experimental MRE data $\mathrm{U}_{0}(\mathrm{y}, \mathrm{z})$ are obtained in 2D Cartesian coordinates and then expressed in polar coordinates U0(r, ) using interpolation. The 1D U (r, $\theta=\mathrm{i}) 0$ experimental data are used to identify the 1D U(r) model for the direction i using the ADbased gradient method described in section 2.1. This process is reiterated for each direction i from $0^{\circ}$ to $350^{\circ}$ with a $10^{\circ}$ step.

The convention used here for the shear modulus $\mu$ as a function of the angle $\theta$ in the coronal $(y, z)$ plane is that $\theta=0$ along the $y$-axis. All these profiles were fitted by a transverse, isotropic model. The anisotropic stiffness profile is therefore estimated using equation (12) to deduce the $\mathrm{c}_{44}$ and $\mathrm{c}_{66}$ parameters as well as the main fiber orientation $\theta 0$ (Royer and Dieulesaint 2000). It is important to note that our method does not make any assumption on the type of anisotropy, as it provides the actual profile $\mu(\theta)$. We have chosen to fit such profiles by a transverse isotropic model since it is one of the most commonly accepted profiles of anisotropy for muscular tissues and it has also been previously shown to be relevant for the anisotropic PVA phantom used in this study (Chatelin et al 2014).

\subsubsection{Application of the method to an isotropic experimental phantom}

The homogeneous isotropic phantom is prepared from a $7 \%$ wt gelatin powder (VWR International, Radnor, PA, USA) diluted in water at $50^{\circ} \mathrm{C}$. After cooling at room temperature, $2 \%$ wt agar-agar powder (AppliChem $\mathrm{GmbH}$, Darmstadt, Germany) is added as scattering particles for the acoustic radiation force generation. An acoustic absorber is placed at the bottom of the phantom to ensure absorption and to avoid unexpected reflections of ultrasound waves, as illustrated in Figure 2 (B). MRE measurements are performed in the phantom as described in subsection 2.3.1. The method described in the subsection 2.2 is applied on the MRE data in order to estimate the anisotropic stiffness profile $\mu^{*}(\theta)$ and to compare it to the theoretical distribution $\mu_{t h}(\theta)$ that corresponds to a transverse isotropic profile.

The mechanical properties of the isotropic gelatin phantom are measured using a rotational rheometer (Thermo Scientific HAAKE MARS III, Rheology Solutions, Bacchus Marsh, Australia). Small cylindrical samples (20 $\mathrm{mm}$ in diameter, $3 \mathrm{~mm}$ in height) are extracted from the phantom for a shear rotating dynamic mechanical analysis (DMA) in a parallel-plate configuration under controlled temperature $\left(22.5^{\circ} \mathrm{C}\right)$. Strain 
sweep experiments are first performed to confirm the linear viscoelastic region (shear strain $\gamma$ from 0.01 to 0.5 at $1 \mathrm{~Hz}$ ), followed by frequency sweep experiments (from 0.1 to $10 \mathrm{~Hz}$ at shear strain $\gamma=0.02$ to ensure testing the sample in the linear domain).

\subsubsection{Application of the method to anisotropic media.}

The method has been successively applied to two different anisotropic media.

2.4.3.1. In an anisotropic tissue-mimicking hydrogel phantom.

A PVA cryogel phantom is prepared (5 \% wt PVA solution (Sigma-Aldrich, St Louis, USA), $1.5 \%$ wt nondissolved agar-agar powder (AppliChem GmbH, Darmstadt, Germany) and degassed distilled water) using a recently-described protocol aimed at inducing mechanical transverse isotropy in a cryogel PVA phantom (Millon et al 2006, Wan et al 2009), following the protocol described by (Chatelin et al 2014). The PVA phantom is embedded in $7 \% \mathrm{wt}$ gelatin, so that the fibers are aligned in the $(\mathrm{y}, \mathrm{z})$ plane (figure $2(\mathrm{C})$ ). An acoustic absorber as well as a $30 \mathrm{~mm}$-thick gelatin hydrogel including agar-agar powder are added at the bottom of the phantom, as described in the previous subsection. MRE measurements are performed in the tissue-mimicking phantom as described in the section 2.2. The identified anisotropic stiffness profile $\mu(-)$ is then compared to the theoretical distribution $\mu \operatorname{th}(\theta)$ that corresponds to a transverse isotropic profile.

2.4.3.2. In an ex vivo biological anisotropic tissue. A fibrous beef back muscle is embedded in $7 \%$ wt gelatin (VWR International, Radnor, PA, USA) so that the muscular fibers are aligned in the $(\mathrm{y}, \mathrm{z})$ coronal plane (figure 2(D)). The muscle is positioned to form an angle of $30^{\circ}$ between the muscular fibers and the y-axis. An acoustic absorber as well as a $30 \mathrm{~mm}$ thick gelatin hydrogel including agar-agar powder are added at the bottom of the phantom, as described in the previous subsection. MRE measurements are performed in the muscle as described in section 2.2. The identified anisotropic stiffness profile $\mu^{*}(\theta)$ is compared to the theoretical distribution $\mu \operatorname{th}(\theta)$ that corresponds to a transverse isotropic profile.

\section{Results}

\subsection{Numerical phantoms}

The harmonic shear wave displacement field obtained for the anisotropic Green formalism with noise as a numerical phantom is shown in figure 3(A). The wave length is found to be higher by a factor of $\sqrt{2}$ in the longitudinal direction when compared to the wavelength in the transverse direction. This is in agreement with the fact that the numerical phantom is twice as stiff in the longitudinal (y-axis) than in the transverse (z-axis) direction.

The stiffness is successively investigated for angles $\theta$ ranging from $0^{\circ}$ to $350^{\circ}$ by $10^{\circ}$ steps using the AD-based gradient method ( $\theta=0^{\circ}$ corresponds to the $y$-axis, i.e. to the longitudinal direction). Less than two seconds are required for the computation time for the stiffness estimation for a single direction. The experimental stiffness profile is represented by blue squares in figure 3(B), with values ranging from $2140 \mathrm{~Pa}$ to $4079 \mathrm{~Pa}$. This profile $\mu *(\theta)$ is very well described by the theoretical selected distribution $\mu \operatorname{th}(\theta)$. Following equation (12), the longitudinal and transverse stiffness values, and the longitudinal direction are estimated to be $\mathrm{c} 44=4053 \pm 116 \mathrm{~Pa}, \mathrm{c} 66=1912 \pm 202 \mathrm{~Pa}$, and $\theta 0=0.02 \pm 1.44^{\circ}$ (orange line), respectively. The error is of $1.33 \%, 4.40 \%$ and $0.006 \%$ for $\mathrm{c} 44$, c66 and $\theta 0$, respectively (root mean square error, $\mathrm{RMSE}=197.9, \mathrm{R} 2=0.949$ ). As predicted by the transverse isotropic model, the stiffness profile has a hippopede shape. The small estimation errors are mainly due to the different Green formalism used for the computation of the numerical phantom and the one used for the resolution of the problem, as well as to the presence of the noise. 


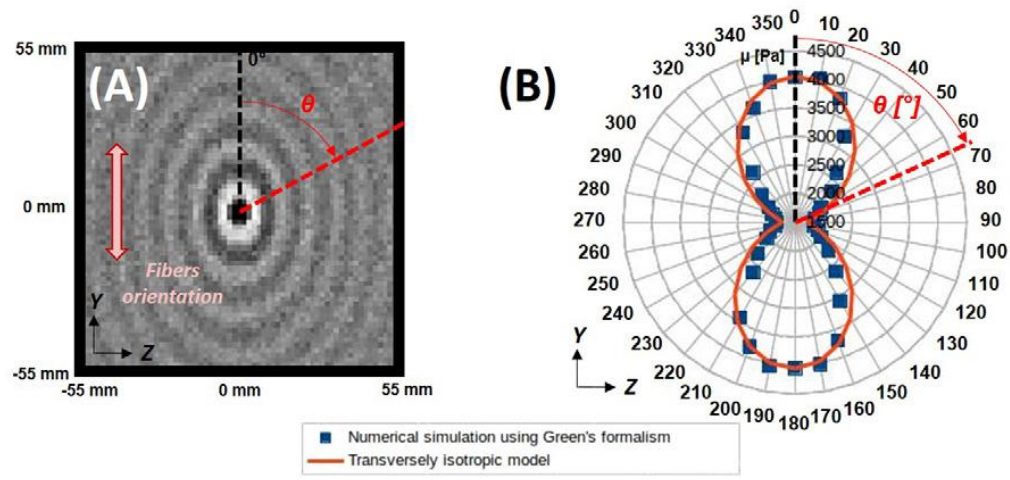

Figure 3. Shear wave front in the numerical anisotropic phantom (A). The stiffness profile $\mu^{*}(\theta)$ is estimated using the AD-based method (B, blue squares). This profile is identified by a theoretical selected transverse isotropic distribution (orange line).

\subsection{Experimental results in the isotropic hydrogel phantom}

The T2-weighted image and the harmonic shear wave displacement field (MRE phase image) obtained by MRE at $100 \mathrm{~Hz}$ in the isotropic gelatin phantom are shown in figures 4(A) and (B), respectively (coronal plane). The stiffness is identified for the angles $\theta$ ranging from $0^{\circ}$ to $350^{\circ}$ by $10^{\circ}$ steps. Less than two seconds are required for the computation time for the stiffness estimation for a single direction. As expected for an isotropic medium, no privileged direction is found. The identified stiffness profile $\mu(-)$ is found to have a mean value of $4449 \pm 343 \mathrm{~Pa}$ over all the directions (figure $4(\mathrm{C})$ ). The identification by the theoretical selected distribution yields $\mathrm{c} 44=4395 \pm 95 \mathrm{~Pa}, \mathrm{c} 66=4309 \pm 86 \mathrm{~Pa}$, and $\theta 0=-4.3 \pm 43.3^{\circ} \quad$ (orange line).

The shear DMA configuration is shown in figure 4(D). The strain sweep tests at $\mathrm{f} 0=1 \mathrm{~Hz}$ frequency allow us to establish a limit higher than $\gamma 0=0.5$ for linear elasticity, assessing that the MRE and frequency sweep DMA experiments are performed in the linear viscoelastic domain of the gelatin phantom. The results from the frequency sweep at $\gamma 0=0.02$ strain are presented in figure 4(E) in terms of the magnitude of the complex modulus $G=\sqrt{ }\left(G^{\prime 2}+(\omega \eta)^{2}\right)$ ) and viscosity $\eta$, assuming a Voigt model ( and $G$ being the pulsation and the storage modulus, respectively). As expected, the dynamic viscosity decreases with frequency. The storage modulus is found to be significantly higher than the loss modulus ( $G^{\prime}>80 * G^{\prime}$ '), which means that the assumption of pure elasticity is valid for this hydrogel. Despite the fact that these results were obtained at different frequency ranges, the comparison between shear stiffness from extrapolated DMA and MRE are in good agreement $\left(\mu_{\text {MRE_100Hz }}=1.08 \times \mu_{\text {DMA_3 }}\right.$ Hz $)$.
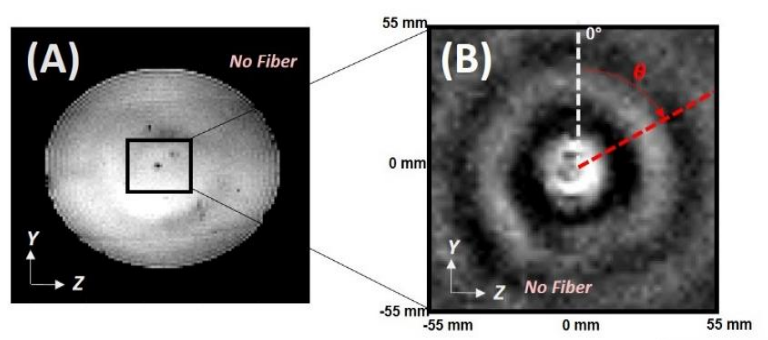

(C)

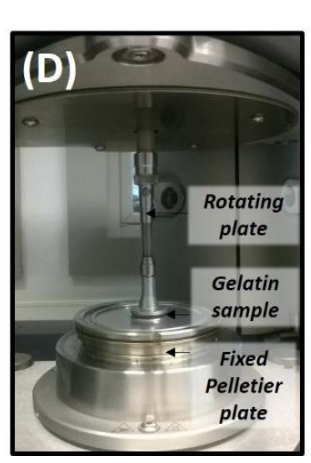

(E)

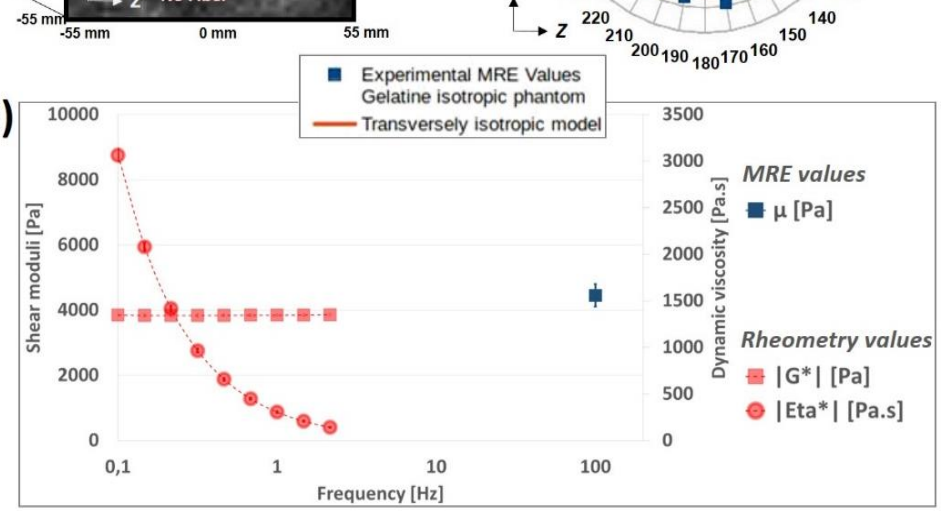

Figure 4. T2-weighted image of the isotropic gelatin phantom in the coronal plane (A). Phase image, in which shear waves are propagating circularly (B). By applying the AD-based method, the stiffness profile $\mu^{*}(\theta)$ is identified (C, blue squares). This profile is identified by a theoretical selected distribution ( $\mathrm{C}$, orange line). The mean mechanical parameters measured by MRE are then compared to classical DMA acquisitions (experimental configuration shown in D) in frequency sweep experiments (E). 


\subsection{Experimental results in the ex vivo muscle}

The results obtained in the two different anisotropic media are presented in this section and illustrated in figure 5 .

3.3.1. Experimental results in the anisotropic PVA phantom. The T2-weighted image and the harmonic shear wave displacement field obtained by MRE (phase image) at $200 \mathrm{~Hz}$ in the PVA anisotropic cryogel phantom are shown in figures 5(A) and (B), respectively (coronal plane). The stiffness is evaluated for angles $\theta$ ranging from $0^{\circ}$ to $350^{\circ}$ by $10^{\circ}$ steps using the AD-based gradient method. The identified profile $\mu(\theta)$ is shown in figure $5(\mathrm{~F})$ (blue squares). Stiffness is found to vary between $4622 \mathrm{kPa}$ and $9855 \mathrm{kPa}$. The identification by the theoretical selected distribution yields estimates of the longitudinal and transverse stiffnesses to $\mathrm{c}_{44}=9037 \pm 483 \mathrm{~Pa}, \mathrm{c}_{66}=4925 \pm 224 \mathrm{~Pa}$ and a longitudinal direction of $\theta_{0}=2.0 \pm 1.4^{\circ}$ (orange line) $(\mathrm{RMSE}=823.0, \mathrm{R} 2=0.773)$.

3.3.2. Experimental results in the ex vivo muscle. The T2-weighted image and the harmonic shear wave displacement field obtained by MRE (phase image) at $100 \mathrm{~Hz}$ in the beef muscle are shown in figures 5(D) and (E), respectively (coronal plane). The stiffness is evaluated for angles $\theta$ ranging from $0^{\circ}$ to $350^{\circ}$ by $10^{\circ}$ steps using the AD-based gradient method. Less than two seconds are required for the computation time for the stiffness estimation for a single direction (with a computation time depending on the initial parameter. The identified profile $\mu(\theta)$ is shown in figure $5(\mathrm{~F})$ (blue squares). Stiffness is found to vary between 3531 $\mathrm{kPa}$ and $9116 \mathrm{kPa}$. The identification by the theoretical selected distribution yields estimates of the longitudinal and transverse stiffness of $\mathrm{c}_{44}=8109 \pm 361 \mathrm{~Pa}, \mathrm{c}_{66}=4166 \pm 292 \mathrm{~Pa}$ and a longitudinal direction $\theta_{0}=-25.6$ $\pm 4.5^{\circ} \quad$ (orange line) $(\mathrm{RMSE}=783.4, \mathrm{R} 2=0.775)$.
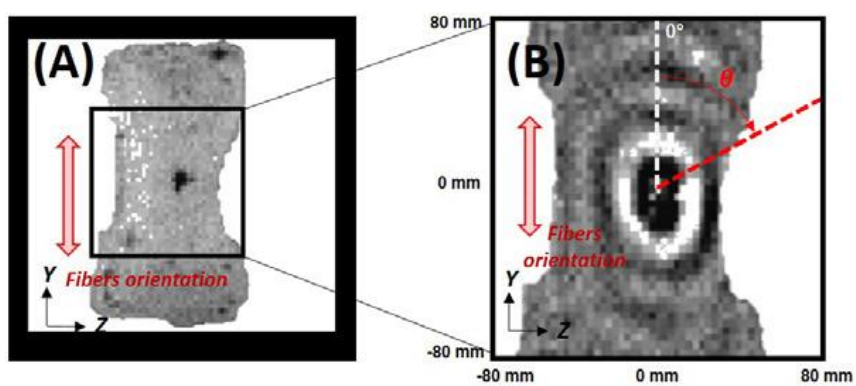

(C)

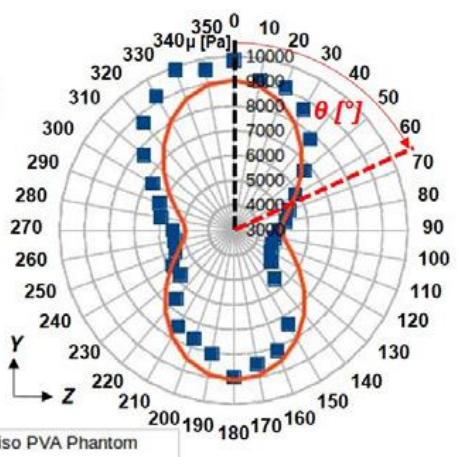

n Experimental MRE Values Aniso PVA Phantom
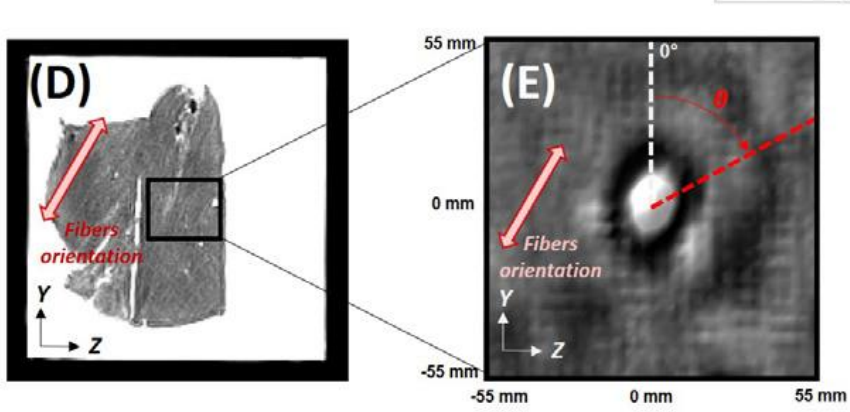

(F)

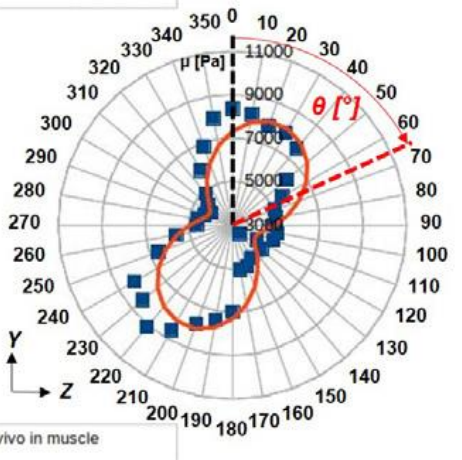

Figure 5. T2-weighted image of the anisotropic PVA phantom (A) and ex vivo beef muscle (D) in the coronal plane. Phase image, in which shear waves propagate circularly ((B) and (E), respectively). The stiffness profile $\mu^{*}(\theta)$ is identified by applying the AD-based method $((\mathrm{C})$ and $(\mathrm{F})$, respectively, blue squares). This profile is compared to a transverse isotropic distribution $((\mathrm{C})$ and $(\mathrm{F})$, respectively, orange line).

\section{Discussion}

Quantitative measurement of in vivo anisotropic mechanical properties is one of the most challenging issues in elastography. By combining a precisely-located shear wave excitation using focalized ultrasound and an AD-based gradient method for specific directions of space, this paper proposes a novel MRE approach for shear wave elastography in fibrous tissues. The method was first assessed on a numerical transverse isotropic 
phantom for stiffness estimation. The method was then tested on an isotropic gelatin phantom and the stiffness estimations were found to be in good agreement with measurements obtained by classical rheometry. The method was also tested in vitro on a fibrous tissue, the mechanical anisotropy of which was evidenced from the stiffness evaluation.

An elastic material with transverse isotropy is described by five independent elastic constants $\left(\mathrm{C}_{11}, \mathrm{C}_{33}, \mathrm{C}_{44}\right.$, $\mathrm{C}_{13}, \mathrm{C}_{66}$ ). While the $\mathrm{C}_{44}$ and $\mathrm{C}_{66}$ parameters are only related to shear wave propagation, the $\mathrm{C}_{11}, \mathrm{C}_{13}$ and $\mathrm{C}_{33}$ parameters are mostly linked to the bulk modulus of the soft tissue (Royer and Dieulesaint 2000). Due to the limited FOV during experimental acquisitions $(300 \mathrm{~mm} \times 300 \mathrm{~mm})$, the propagation of the compression wave (with wave lengths of several meters) is generally ignored. This limitation is common to most of the current dynamic elastography techniques. Since an estimation of the $C_{11}$ coefficient is necessary, the material was supposed to be nearly incompressible and the propagation velocity of $1500 \mathrm{~m} \cdot \mathrm{s}^{-1}$ was used to fix the $\mathrm{C}_{11}$ parameter to $2.25 \mathrm{GPa}$. The identification process is thus focused on the $\mathrm{C}_{44}$ and $\mathrm{C}_{66}$ parameters only.

As observed in previous studies, the use of a gradient method for elasticity inversion using a least-squares formulation increases the robustness and decreases the sensitivity to the information loss (such as noise) in comparison to common inversion methods (Arnal et al 2013). The originality of our approach is to use a gradient method for elasticity inversion coupled with an AD method and L-BFGS-B optimizer in one dimension for specific directions for the characterization of tissue anisotropy. The low computation time is one of the significant advantages of the method. It allows the identification of a large number of parameters using a limited amount of computer memory and, consequently, time. In our study, only one parameter of a simple 1D Green model was identified per direction, since tissue was considered to be purely elastic (one stiffness value $\mu$ per direction $\theta$ ). The advantages of using an AD and a L-BFGS-B optimization process over a conventional approach are not significant in such a simple case. However, this study demonstrates the potential of using such an approach to evaluate mechanical parameters and pave the way to the simultaneous identification of a large number of parameters of more complex models of wave propagation. For instance, the identification of isotropic analytical models, heterogeneous finite difference models (FDMs), finite element models (FEMs) (Rouze et al 2013, Qiang et al 2015), and viscoelastic parameters are part of our ongoing research.

The comparison between the mean stiffness measured by DMA and the one measured by the proposed method in the gelatin phantom confirms the ability of the method to identify elasticity in a quantitative manner (figure 4(E)). However, due to technological limitations, the frequency range investigated using DMA cannot be extended to the MRE frequency and the assessment of the values can only be carried out by extrapolating the results from the frequency sweep experiments. The variations in the measurement of $\mu(\theta)$ in the isotropic phantom (figure 4(C)) can be explained either by the presence of noise in the MRE images, by heterogeneities in the gelatin phantom or by spatial irregularities in the generation of the shear waves. The tangent linear code generated with $\mathrm{AD}$ allows for gradient computations at evaluation time. Data are thought to be impacted by the Gaussian noise resulting from MR image acquisition and processing. Gaussian noise is added on the data, which is relevant to the proposed least squares method. Convergence is also improved by identifying unknown parameters, one for each angle, and by the use of the identified value as an initial guess for the optimization with the next angle. We have demonstrated the ability of the method to identify the anisotropic profile both in the transversely isotropic phantom that has been characterized in the literature (Chatelin et al 2014) and in the anisotropic beef muscle tissue in vitro.

Our gradient method is currently limited to anisotropic uniform media under the assumption of local homogeneity around the focal zone. The presented unidirectional Green formalism does not allow the identification of mechanical properties in a non-uniform medium. This limitation can be overcome by including heterogeneous mechanical parameters and by using a different shear wave propagation model, for instance a FEM or FDM of shear wave propagation in a heterogeneous medium (Rouze et al 2013, Qiang et al 2015). This would extend the scope of the proposed method to anisotropic, heterogeneous media.

The application to the evaluation of anisotropic stiffness is made possible by the use of a circular wave whose origin is precisely identified. Currently, two techniques allow us to generate point-source circular shear waves. As proposed in 2006 by Chan et al and afterwards adapted for real-time MRE in interventional radiology, the first approach consists of using a vibrating needle inserted in the tissue (Chen et al 2006, 2014, Zhao et al 2008, Corbin et al 2015). In this study, we use a noninvasive approach, which consists of the use of 
an acoustic radiation force. As proposed by Wu et al (2000) and Souchon et al (2008), its synchronization with the MRE sequences provides an imaging of the shear wave field, as shown in figures $4(B)$ and $5(B)$ and (E) (phase images).

The shear wave is pseudo-harmonic, but it would be also possible to generate transient circular shear waves, to visualize them using MRI and to identify the mechanical properties using the impulse Green formalism (Bercoff et al 2004, Chatelin et al 2015). As shown in figures 3(B) and 5(C) and (F), the stiffness profile obtained by our method in a transverse isotropic medium is similar to a theoretical selected distribution (Royer and Dieulesaint 2000, Wang et al 2013), with higher stiffness in the longitudinal than in the transverse direction. It is worth noting that our method is not restricted to transverse isotropy. While solving directly the inverse problem in two dimensions in Cartesian coordinates would involve assumptions about the sort of anisotropy (for instance transverse isotropy) in the forward model, the identification by our method allows us to identify a stiffness profile without any assumption about the nature of anisotropy. While the transverse isotropic model is most commonly adopted for the muscles in the literature (Gennisson et al 2003, 2005, 2010, Bensamoun et al 2006, Ringleb et al 2007, Namani et al 2009, Green et al 2013, Qin et al 2013, Chatelin et al 2015), it is difficult to certify that the muscular fibers are oriented all through the sample. We have shown that the transverse isotropic model is well suited for the PVA phantom. The variation obtained in the identification of the transverse isotropic profile in the muscle can be explained by the complex fibrous architecture of the tissue resulting in spatial variation in the main fiber direction. Our approach is limited to a bi-dimensional investigation of the mechanical anisotropy since we assume the muscular fibers are aligned with the imaging plane. The anisotropy evaluation is restricted to bi-dimensional anisotropy tensors (i.e. the transverse isotropic tensors restricted either to the focal plane or to a plane parallel to the focal plane (y, z) of the ultrasonic probe).

In this study, the application of the AD-based method has been limited to the investigation of the anisotropic stiffness, under the assumption of pure elasticity. The influence of the viscosity was neglected. Consequently, these parameters can be identified independently without any link with the convergence or stability of the identification process (Charpentier and Roux 2004). Identifying the viscosity in addition to the stiffness will not result in any modification of convergence or stability compared to the identification of the stiffness only. The identification process for these two parameters can be done either independently or hierarchically in the case of highly viscous tissue $\left(G^{\prime \prime}\right.$ higher than five to ten times $G^{\prime}$ ) by adapting the cost function. In any case, the priority will be given to the identification of the stiffness. At this point, the identification of the viscosity requires improving the phase-to-noise ratio of our experimental MRE data. The next steps of this study will then consist in applying and validating the AD-based gradient method for the evaluation of both stiffness and viscosity profiles in either anisotropic analytical (Vavryčuk 2007, Chatelin et al 2015) or FEM-based (Rouze et al 2013, Qiang et al 2015) numerical phantoms. Some previous studies have proposed preliminary approaches in this way (Manduca et al 2001, Arnal et al 2013). The proposed method was originally developed to provide reliable in vivo, preclinical quantitative measurements on animals. Extension of this method to a clinical use requires thorough investigation of the specifications of our excitation device. It would be necessary to develop and calibrate (with respect to Food and Drug Administration (FDA) requirements) specific MRcompatible ultrasound probes, and careful measurement of the pressures and temperatures generated by the ultrasonic probe will be necessary to ensure compliance with FDA requirements in terms of mechanical index, spatial-peak time average intensity (ISPTA) and thermistance. The use of a gradient-based data assimilation together with an AD-based method shows a great potential for MRE reconstructions. This method exhibits substantial benefits for quantitative investigations of complex mechanical properties, such as anisotropy, and opens up numerous perspectives both for clinical use and for in vivo biomechanical testing.

\section{Acknowledgement}

This work was supported by French state funds managed by the ANR (Agence Nationale de la Recherche) within the Investissements d'Avenir program for the Labex CAMI (Computer Assisted Medical Intervention, ANR-11-LABX-004) and the IHU Strasbourg (Institut Hospitalo-Universitaire, Institute of Image Guided Surgery, ANR-10-IAHU-02).

\section{Appendix}

The medium is assumed to be semi-infinite, anisotropic, elastic and homogeneous. The stiffness tensor $\boldsymbol{C}$ is required to locally describe the stiffness along each direction of the space and is defined by:

$$
\boldsymbol{\sigma}=\mathbf{C} \boldsymbol{\varepsilon}
$$


where $\boldsymbol{\sigma}$ and $\boldsymbol{\varepsilon}$ are the stress and strain tensors respectively. In the specific case of transverse isotropy, the representative matrix of the Christoffel stiffness tensor can be simplified by using the Voigt notation as:

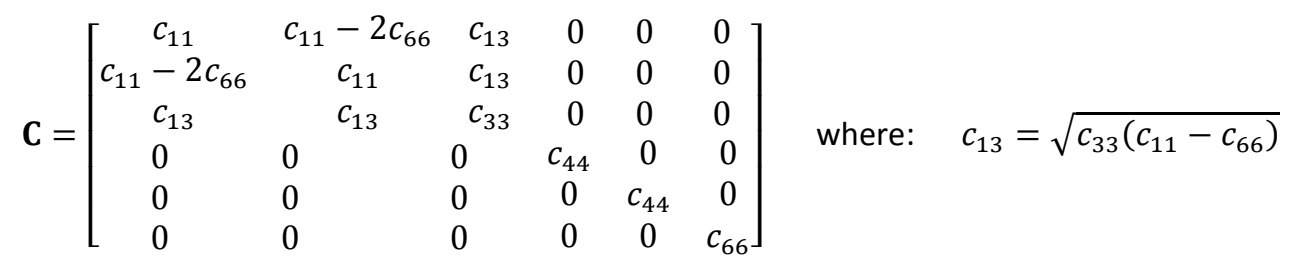

The calculation of the displacement vector from one point source in the temporal domain is expressed using the transversely isotropic elastic Green formalism in Equation A1. The terms $G_{k l}^{(P)}, G_{k l}^{(S V)}, G_{k l}^{(S H)}, G_{k l}^{(S V-S H)}$ and $G_{k l}^{(P-S V)}$ correspond to the contribution of the P-wave, SV-wave, SH-wave, near-field term between Pwave and SV-wave and near-field term between P-wave and SH-wave, respectively (Equation A4 to A6). For more details on these coefficients, the reader is referred to [Vavryčuk 2001]).

$$
U_{\mathrm{k}}(\mathbf{x}, \mathrm{t})=\sum_{l} \frac{F_{l}}{4 \pi \rho}\left\{G_{k l}^{(P)}(\mathbf{x}, \mathrm{t})+G_{k l}^{(S V)}(\mathbf{x}, \mathrm{t})+G_{k l}^{(S H)}(\mathbf{x}, \mathrm{t})+G_{k l}^{(S V-S H)}(\mathbf{x}, \mathrm{t})+G_{k l}^{(P-S V)}(\mathbf{x}, \mathrm{t})\right\}
$$

where $t$ is the time, $\mathbf{x}=(\mathrm{x}, \mathrm{y}, \mathrm{z})$ the Cartesian coordinates of specific observation point, $\rho$ the density and $(k, l)$ the direction index numbers of the displacement and the source, respectively.

The other parameters of the exact formula for the Green functions are:

$$
\begin{aligned}
& G_{k l}^{(P)}(\mathbf{x}, \mathrm{t})=\frac{g_{k}^{(1)} g_{l}^{(1)}}{\tau^{(1)} \sqrt{c_{11}^{3}}} \delta\left(t-\tau^{(1)}\right) \\
& G_{k l}^{(S V)}(\mathbf{x}, \mathrm{t})=\frac{g_{k}^{(2)} g_{l}^{(2)}}{\tau^{(2)} \sqrt{c_{44}^{3}}} \delta\left(t-\tau^{(2)}\right), \\
& G_{k l}^{(S H)}(\mathbf{x}, \mathrm{t})=\frac{g_{k}^{(3)} g_{l}^{(3)}}{\tau^{(3)} c_{66} \sqrt{c_{44}}} \delta\left(t-\tau^{(3)}\right), \\
& G_{k l}^{(S V-S H)}(\mathbf{x}, \mathrm{t})=\frac{1}{\sqrt{c_{44}}} \frac{g_{k}^{(3) \perp} g_{l}^{(3) \perp}-g_{k}^{(3)} g_{l}^{(3)}}{R^{2}} \int_{\tau^{(2)}}^{\tau^{(3)}} \delta(t-\tau) d \tau, \\
& G_{k l}^{(P-S V)}(\mathbf{x}, \mathrm{t})=\frac{3 g_{k}^{(1)} g_{l}^{(1)}-\delta_{k l}}{r^{3}} \int_{\tau^{(1)}}^{\tau^{(2)}} r \delta(t-\tau) d \tau .
\end{aligned}
$$

The travel times of the compressional P-, shear SV- (i.e. parallel to the fibers) and shear SH- (i.e. orthogonal to the fibers) wave are respectively defined by:

$$
\tau^{(1)}=\frac{r}{\sqrt{c_{11}}}, \quad \tau^{(2)}=\frac{r}{\sqrt{c_{44}}}, \quad \tau^{(3)}=\frac{r}{\sqrt{c_{66}}} \sqrt{N_{1}^{2}+N_{2}^{2}+\frac{c_{66}}{c_{44}} N_{3}^{2}} .
$$

The polarization vectors are defined by:

$$
g^{(1)}=\left[\begin{array}{c}
N_{1} \\
N_{2} \\
N_{3}
\end{array}\right], \quad g^{(2)}=\frac{1}{\sqrt{N_{1}^{2}+N_{2}^{2}}}\left[\begin{array}{c}
-N_{1} N_{3} \\
-N_{2} N_{3} \\
N_{1}^{2}+N_{2}^{2}
\end{array}\right], \quad g^{(3)}=\frac{1}{\sqrt{N_{1}^{2}+N_{2}^{2}}}\left[\begin{array}{c}
N_{2} \\
-N_{1} \\
0
\end{array}\right], \quad g^{(3) \perp}=\frac{1}{\sqrt{N_{1}^{2}+N_{2}^{2}}}\left[\begin{array}{c}
N_{1} \\
N_{2} \\
0
\end{array}\right] \text {. }
$$

where $\mathrm{r}=\sqrt{\mathrm{x}_{1}^{2}+\mathrm{x}_{2}^{2}+\mathrm{x}_{3}^{2}}$ and $\mathrm{R}=\sqrt{\mathrm{x}_{1}^{2}+\mathrm{x}_{2}^{2}}$ are the distance from the source point to the observation point and the distance from the focal axis to the observation point, respectively. $N_{m}=x_{m} / r$ is the unit direction vector from the source point to the observation point. $c_{11}=\rho c_{P}^{2}, c_{44}=\rho c_{S V}^{2}$ and $c_{66}=\rho c_{S H}^{2}$ are the coefficients 
of the Christoffel stiffness tensor $\mathbf{C}\left(c_{P}, c_{S V}\right.$ and $c_{S H}$ correspond to the celerity of the P-wave, SV-wave and SH-wave, respectively).

\section{References}

[1] Aki, L. and Richards, P. G. (1980). Quantitative Seismology, Theory and Methods 1, chap 4, New York: W. H. Freeman and Co.

[2] Amador C., urban M.W., Chen S. and Greenleaf J.F. (2011). Shearwave dispersion ultrasound vibrometry (sduv) on swine kidney. IEEE Transactions on Ultrasonics, Ferroelectrics, and Frequency Control 58(12), pp.2608-2619.

[3] Arnal, B., Pinton, G., Garapon, P., Pernot, M., Fink, M. and Tanter, M. (2013). Global approach for transient shear wave inversion based on the adjoint method: a comprehensive 2D simulation study. Physics in Medicine and Biology 58, pp.6765-6778.

[4] Arndt R., Schmidt S., Loddenkemper C., Grünbaum M., Zidek W., Van der Giet M. and Westhoff T.H. (2010). Noninvasive evaluation of renal allograft fibrosis by transient elastography-A pilot study. Transpl Int 23, pp.871-877.

[5] Basford J.R., Jenkyn T.R., An K.-N., Ehman R.L., Heers G. and Kaufman K.R. (2002). Evaluation of healthy and diseased muscle with magnetic resonance elastography. Arch Phys Med Rehabil 83, pp.1530-1536.

[6] Bensamoun, S.F., Ringleb, S.I., Littrell, L., Chen, Q., Brennan, M., Ehman, R.L. and An, K.-N. (2006). Determination of thigh muscle stiffness using magnetic resonance elastography. Journal of the Magnetic Resonance Imaging 23(2), pp.242-247.

[7] Bensamoun, S.F., Ringleb, S.I., Chen, Q., Ehman, R.L., An, K.-N. and Brennan, M. (2007). Thigh muscle stiffness assessed with magnetic resonance elastography in hyperthyroid patients before and after medical treatment. J. of Magnetic Resonance Imaging 26(3), pp.708-713.

[8] Bercoff, J., Tanter, M. and Fink, M. (2004). Supersonic shear imaging: a new technique for soft tissue elasticity mapping. IEEE Trans. Ultrason. Ferroelectr. Freq. Control 51, pp.396-409.

[9] Brum, J., Bernal, M., Gennisson, J.-L., Tanter, M. (2013). In vivo evaluation of the elastic anisotropy of human Achilles tendon using shear wave dispersion analysis. Physics in Medicine and Biology 55, pp.505-523.

[10] Catheline, S., Gennisson, J.-L., Delon, G., Fink, M., Sinkus, R., Abouelkaram, S. and Culioli, J. (2004). Measurement of viscoelastic properties of homogeneous soft solid using transient elastography: an inverse problem approach J. Acoust. Soc. Am. 116, pp.3734-41.

[11] Chan, Q.C., Li, G., Ehman, R.L., Grimm, R.C., Li, R. and Yang E.S. (2006). Needle shear wave driver for magnetic resonance elastography. Magn Reson Med 55, pp.1175-1179.

[12] Charpentier, I. and Dal Cappello, C. (2015). Higher-order automatic differentiation of mathematical functions. Computer Physics Communications 189, pp.66-71.

[13] Charpentier I and Roux P 2004 Mode and wavenumber inversion in shallow water using an adjoint method J. Comp. Acous. $12521-42$

[14] Chatelin, S., Gennisson, J.-L., Bernal, M., Tanter, M. and Pernot, M. (2015). Modelling the impulse diffraction field of shear waves in transverse isotropic viscoelastic medium. Physics in Medicine and Biology 60, pp.3639-3654.

[15] Chatelin, S., Bernal, M., Deffieux, T., Papadacci, C., Flaud, P., Nahas, A., Boccara, C., Gennisson, J.-L., Tanter, M. and Pernot, M. (2014). Anisotropic polyvinyl alcohol hydrogel phantom for shear wave elastography in fibrous biological soft tissue: a multimodality characterization. Physics in Medicine and Biology 59, pp.6923-6940.

[16] Chen, J., Woodrum, D.A., Glaser, K.J., Murphy, M.C., Gorny, K. and Ehman, R. (2014). Assessment of in vivo laser ablation using MR elastography with an inertial driver. Magn Reson Med 72, pp.59-67.

[17] Corbin, N., Vappou, J., Breton, E., Boehler, Q., Barbé, L., Renaud, P. and de Mathelin, M. (2015). Interventional MR elastography for MRI-guided percutaneous procedures. Magnetic Resonance in Medicine, doi: 10.1002/mrm.25694.

[18] Correia, M., Chatelin, S., Papadacci, C., Provost, J., Villemain, O., Tanter, M. and Pernot, M. (2014). Wave Ultrafast Harmonic Compounding for cardiac shear wave imaging. Proc. Of the IEEE International Ultrasonics Symposium, Chicago, US.

[19] Couade, M., Pernot, M., Messas, E., Bel, A., Ba, M., Hagège, A., Fink, M. and Tanter, M. (2011). In Vivo Quantitative Mapping of Myocardial Stiffening and Transmural Anisotropy during the Cardiac Cycle. IEEE Transactions on Medical Imaging 30(2), pp.295-305.

[20] Fatemi, M., Greenleaf, J. (1998). Ultrasound-stimulated vibro-acoustic spectrography. Science 280, pp.82-85.

[21] Fung, Y.C., 1993. Biomechanics: mechanical properties of living tissues. New York: Springer-Verlag.

[22] Gennisson, J.-L., Catheline, S., Chaffaï, S., Fink, M., 2003. Transient elastography in anisotropic medium: application to the measurement of slow and fast shear wave speeds in muscles. J. Acoust. Am. 114(1), pp.536-541

[23] Gennisson, J.-L., Cornu, C., Catheline, S., Fink, M. and Portero, P. (2005). Human muscle hardness assessment during incremental isometric contraction using transient elastograph. Journal of Biomechanics 38(7), pp.1543-1550.

[24] Gennisson, J.-L., Deffieux, T., Macé, E., Montaldo, G., Fink, M., Tanter, M. (2010). Viscoelastic and anisotropic 
mechanical properties of in vivo muscle tissue assessed by supersonic shear imaging, Ultrasound in medicine \& biology 36(5), pp.789-801.

[25] Gennisson J.-L., Grenier N., Combe C. and Tanter M. (2012). Supersonic Shear Wave Elastography of In Vivo Pig Kidney: Influence of Blood Pressure, Urinary Pressure and Tissue Anisotropy. Ultrasound in Medicine and Biology 38(9), pp.1559-1567.

[26] Green, M., Geng, G., Qin, E., Sinkus, R., Gandevia, S.C. and Bilston, L.E. (2013), Measuring anisotropic muscle stiffness properties using elastography, NMR in Biomed 26, pp.1387-1394.

[27] Griewank, A. and Walther, A. (2008). Evaluating Derivatives: Principles and Techniques of Algorithmic Differentiation, $2^{\text {nd }}$ edition, SIAM, Philadelphia.

[28] Gudbjartsson, H. and Patz, S. (1995). The Rician distribution of noisy MRI data. Magn. Reson. Med. 34(6), pp.910914.

[29] Hascoët, L. and Pascual, V. (2004). TAPENADE 3.1 user's guide, INRIA report RT-0300.

[30] Klatt, D., Papazoglou, S., Braun, J. and Sack, I. (2010). Viscoelasticity-based MR elastography of skeletal muscle. Physics in Medicine and Biology 55(21), pp.6445-6459.

[31] Knutsson, H., Westin, C.-F. and Granlund, G. (1994). Local multiscale frequency and bandwidth estimation. Image processing. Proc IEEE Int Conf 1, pp.36-40.

[32] Lee, W. N., Pernot, M., Couade, M., Messas, E., Bruneval, P., Bel, A., Tanter, M. (2012). Mapping myocardial fiber orientation using echocardiography-based shear wave imaging. Medical Imaging, IEEE Transactions on Medical Imaging, 31(3), pp.554-562.

[33] Manduca, A., Muthupillai, R., Rossman, P.J., Greenleaf, J.F. and Ehman R.L. (1996). Proc. SPIE 2710, Medical Imaging 1996: Image Processing, pp.616.

[34] Manduca, A., Oliphant, T.E., Dresner, M.A., Mahowald, J.L., Kruse, S.A., Amromin, E., Felmlee, J.P., Greenleaf, J.F. and Ehman, R.L. (2001). Magnetic resonance elastography: Non-invasive mapping of tissue elasticity, Med Image Anal 5(4), pp.237-254.

[35] McCullough, M.B., Domire, Z.J., Reed, A.M., Amin, S., Ytterberg, S.R., Chen, Q. and An, K.-N. (2011). Evaluation of muscles affected by myositis using magnetic resonance elastography. Muscle and Nerve 43(4), pp.585-590.

[36] Millon L E, Mohammadi H and Wan W K 2006 Anisotropic polyvinyl alcohol hydrogel for cardiovascular applications J. Biomed. Mater. Res. B 79 305-11

[37] Muthupillai, R., Lomas, D.J., Rossman, P.J., Greenleaf, J.F., Manduca, A. and Ehman R.L. (1995). Magnetic resonance elastography by direct visualization of propagating acoustic strain waves. Science 29, 269(5232), p.18541857.

[38] Namani, R., Wood, M.D., Sakiyama-Elbert, S.E. and Bayly, P.V. (2009). Anisotropic Mechanical Properties of Magnetically Aligned Fibrin Gels Measured by Magnetic Resonance Elastography. J Biomech 42(13), p.2047-2053.

[39] Oberai, A.A., Gokhale, N.H. and Feijoo, R. (2003). Solution of inverse problems in elasticity imaging using the adjoint method. Inverse Problems 19, pp.297-313.

[40] Oliphant, T.E., Manduca, A., Ehman, R.L. and Greenleaf, J.F. (2001). Complex-valued stiffness reconstruction for magnetic resonance elastography by algebraic inversion of the differential equation. Magnetic Resonance in Medicine 45(2), pp.299-310.

[41] Papazoglou, S., Rump, J., Braun, J. and Sack, I. (2006). Shear wave group velocity inversion in MR elastography of human skeletal muscle. Magnetic Resonance in Medicine 56(3), pp.489-497.

[42] Qin, E., Sinkus, R., Geng, G., Cheng, S., Green, M., Rae, C., Bilston, L.E., 2013. Combining MR Elastography and Diffusion Tensor Imaging for the Assessment of Anisotropic Mechanical Properties: A Phantom Study. J. of MRI 37, pp.217-226.

[43] Qiang B, Brigham J C, Aristizabal A, Greenleaf J F, Zhang X and Urban M W 2015 Modeling transversely isotropic, viscoelastic, incompressible tissue-like materials with application in ultrasound shear wave elastography Phys. Med. Biol. 60 1289-306

[44] Ringleb, S.I., Bensamoun, S.F., Chen, Q., Manduca, A., An, K.-N. and Ehman, R.L. (2007). Applications of Magnetic Resonance Elastography to Healthy and Pathologic Skeletal Muscle. Journal of Magnetic Resonance Imaging 25, pp.301-309.

[45] Rouze N C, Wang M H, Palmeri M L and Nightingale K R 2013 Finite element modeling of impulsive excitation and shear wave propagation in an incompressible, transversely isotropic medium J. Biomech. 46 2761-8

[46] Royer, D. and Dieulesaint, E. (2000). Elastic Waves in Solids. New York: Springer.

[47] Sack, I., Rump, J., Elgeti, T., Samani, A. and Braun, J. (2009). MR Elastography of the Human Heart: Noninvasive Assessment of Myocardial Elasticity Changes by Shear Wave Amplitude Variations. Magnetic Resonance in Medicine 61, pp.668-677.

[48] Sandrin, L., Fourquet, B., Hasquenoph, J.-M., Yon, S., Fournier, C., Mal, F., Christidis, C., Ziol, M., Poulet, B., 
Kazemi, F., Beaugrand, M. and Palau, R. (2003). Transient elastography: a new noninvasive method for assessment of hepatic fibrosis. Ultrasound in Medicine and Biology 29(12), pp.1705-1713.

[49] Sarvazyan, A.P., Rudenko, O.V., Swanson, S.D., Fowlkes, J.B., Emelianov, S.Y. (1998). Shear wave elasticity imaging - A new ultrasonic technology of medical diagnostic. Ultrasound Med. Biol., vol. 20, pp.1419-1436.

[50] Shah N.S., Kruse S.A., Lager D.J., Farell-Baril G., lieske J.C., King B.F. and Ehman R.L. (2004). Evaluation of renal parenchymal disease in a rat model with magnetic resonance elastography. Magnetic Resonance in Medicine 52(1), pp. 56-64.

[51] Shcherbakova, D., Papadacci, C., Swillens, A., Caenen, A., De Bock, S., Saey, V., Chiers, K., Tanter, M., Greenwald, S., Pernot, M. and Segers, P. (2014). Supersonic shear wave imaging to assess arterial nonlinear behavior and anisotropy: proof of principle via ex vivo testing of the horse aorta. Advances in Mechanical Engineering, pp.1-12.

[52] Sinkus, R., Tanter, M., Xydeas, T., Catheline, S., Bercoff, J. and Fink, M. (2005). Viscoelastic shear properties of in vivo breast lesions measured by MR elastography. Magnetic Resonance Imaging 23(2), pp.159-165.

[53] Song, P., Zhao, H., Urban, M.W., manduca, A., Pislaru, S.V., Kinnick, R.R., Pislaru, C., Greenleaf, J.F. and Chen, C. (2013). Improved Shear Wave Motion Detection Using Pulse-Inversion Harmonic Imaging with a Phased Array Transducer. IEEE Transaction in Medical Imaging 32(12), pp.2299-2310.

[54] Souchon, R., Salomir, R., Beuf, O., Milot, L., Grenier, D., Lyonnet, D., Chapelon, J.-Y. and Rouvière, O. (2008). Transient MR elastography (t-MRE) using ultrasound radiation force: Theory, safety, and initial experiments in vitro. Magnetic Resonance in Medicine 60(4), pp.871-881.

[55] Van Houten, E., Paulsen, K.D., Miga, M.I., Kennedy, F.E. and Weaver, J.B. (1999). An overlapping subzone technique for MR based elastic property reconstruction. Magn Reson Med 42, pp.779-786.

[56] Vappou, J., (2012). Magnetic Resonance and Ultrasound Imaging-Based Elasticity Imaging Methods : A review. Biomedical Engineering 40(2), pp.121-134.

[57] Vavryčuk, V. (2007). Asymptotic Green's function in homogeneous anisotropic viscoelastic media. Proc. Royal Soc. Am. 463, pp.2689-2707.

[58] Vavryčuk, V. (2001). Exact elastodynamic Green's functions for simple types of anisotropy derived from higherorder ray theory. Stud. Geophys. Geod. 45, pp.67-84.

[59] Wan W, Millon L E and Mohammadi H 2009 Anisotropic hydrogels United States Patent Application Publication US 2009/0214623 A1

[60] Wang, M., Byram, B., Palmeri, M., Rouze, N., Nightingale, K. (2013). Imaging transverse isotropic properties of muscle by monitoring acoustic radiation force induced shear waves using a 2-D matrix ultrasound array. IEEE Transactions on Medical Imaging 32, pp.1671-1684.

[61] Wang, Z.G., Liu, Y., Wang, G.and Sun, L.Z. (2009). Elastography method for reconstruction of nonlinear breast tissue properties. Journal of Biomedical Imaging: ID 406854, 9 pages.

[62] Wu, T., Felmlee, J.P., Greenleaf, J.F., Riederer, S.J. and Ehman, R.L. (2000). MR imaging of shear waves generated by focused ultrasound. Magnetic Resonance in Medicine 43(1), pp.111-115.

[63] Zhao, X.G., Zheng, Y., Liang, J.M., Chan, Q.C., Yang, X.F., Li, G. and Yang, E.S (2008). In vivo tumor detection on rabbit with biopsy needle as MRE driver. Conf Proc IEEE Eng Med Biol Soc, pp.121-124.

[64] Zhu, C., Byrd, R.H., Lu, P. and Nocedal, J. (1997). L-BFGS-B: Fortran subroutines for large-scale bound-constrained optimization. ACM Transactions on Mathematical Software (TOMS) 23(4), pp.550-560. 